\title{
Guest Editorial: Environmental Multimedia Retrieval
}

\author{
Stefanos Vrochidis ${ }^{1} \cdot$ Kostas Karatzas $^{2}$. \\ Ari Karppinen $^{3} \cdot$ Alexis Joly $^{4}$
}

Published online: 28 January 2016

(C) Springer Science+Business Media New York 2016

The rapid advancements of digital technologies, as well as the progress and wide availability of digital cameras and sensors have resulted in a great increase of multimedia data production worldwide. This is also the case for multimedia data that describe the state of the environment, which include huge amounts of data streams from model systems, dedicated stations and amateur sensors, as well as visual environmental information, such as heatmaps and forest satellite images. In parallel, the success of citizen sciences and social networking tools has fostered the emergence of large and structured communities of nature observers (e.g., e-bird, Tela Botanica, etc.), who started to produce outstanding collections of biodiversity multimedia records. Citizens have become increasingly aware of the important role that environmental data (e.g., weather forecast, air quality, life species distributions) play on health issues (e.g., allergies), as well as to a variety of other human activities (e.g., agriculture, trip planning). In

Stefanos Vrochidis

stefanos@iti.gr

Kostas Karatzas

kkara@eng.auth.gr

Ari Karppinen

Ari.Karppinen@fmi.fi

Alexis Joly

alexis.joly@inria.fr

1 Centre for Research and Technology Hellas, Information Technologies Institute, Thessaloniki, Greece

2 Aristotle University, Thessaloniki, Greece

3 Finnish Meteorological Institute, Helsinki, Finland

4 INRIA, Sophia-Antipolis, France 
addition, such data are very important for understanding environmental issues and phenomena, such as the greenhouse effect, global warming and climate change.

Therefore, there is an increasing need for the development of advanced techniques for analysing, interpreting and aggregating environmental data provided in multimedia formats. This will allow for the generation of reliable measurements, as well as for the development of personalised applications that will take into account the state of the environment and the personal health conditions and preferences [5]. Furthermore, it should be noted that the production of accurate and timely knowledge of other living species is essential for a sustainable development of humanity and for biodiversity conservation.

Despite the fact that a large number of multimedia analysis techniques has been developed specifically for extracting events and behaviours in human-centered and general purpose applications, such as sports, movies, surveillance, relatively little attention has been paid to the analysis, retrieval and interpretation of environmental information from multimedia content. Only very recent projects such as $\mathrm{PESCaDO}^{1} \mathrm{Pl} @$ ntNet $^{2}$ and $\mathrm{PASODOBLE}^{3}$ have dealt with developing innovative services that take into account environmental information and investigated the extraction and semantic interpretation of the environmental information encoded in multimedia format such as weather, air quality, pollen forecasts or citizen's multimedia records. In this context, recent works in multimedia analysis of environmental data deal with heatmap analysis for forecast data extraction [3], plant identification [2], underwater visual data analysis [4] and monitoring of the atmosphere [1].

In this context this thematic issue focuses on the analysis, processing, indexing, retrieval and fusion of meteorological data encoded in multimedia, on classification and recognition of plants and biological species for environmental multimedia, as well as on environmental applications. The special issue consists of 7 papers.

The first paper deals with "Focussed Crawling of Environmental Web Resources Based on the Combination of Multimedia Evidence" (10.1007/s11042-015-2624-3). This work proposes a classifier-guided focussed crawling approach that estimates the relevance of a hyperlink to an unvisited Web resource based on the combination of textual and visual evidence. The proposed focussed crawling approach is applied towards the discovery of environmental Web resources that provide air quality measurements and forecasts.

Then, the issue includes a work that focuses on "Environmental data extraction from heatmaps using the AirMerge system" (10.1007/s11042-015-2604-7). The AirMerge platform allows data from multiple heterogeneous chemical weather data sources to be continuously collected in a unified repository. The results demonstrate the potential of this approach for being applied also in other areas, in which image-based environmental information retrieval will be needed.

Another very challenging task in environmental multimedia retrieval research is plant species identification. In this context, "Semantic-based automatic structuring of leaf images for advanced plant species identification" (10.1007/s11042-015-2603-8) is proposed. First, a structuring process is performed by defining geometric parameters based on the analysis of botanical definitions. Then, the species recognition process is based on texture or contour descriptors followed by a KNN classifier. Experiments are carried out using the Scan Pictures of the ImageCLEF 2011 dataset.

\footnotetext{
${ }^{1}$ http://www.pescado-project.eu/

2 http://plantnet-project.org/

${ }^{3}$ http://www.myair.eu/
} 
In this context, we also present "Plant identification: Man vs. Machine On beyond LifeCLEF 2014 plant identification challenge" (10.1007/s11042-015-2607-4). This paper reports a largescale experiment aimed at evaluating how state-of-art computer vision systems perform in identifying plants compared to human expertise. A subset of the dataset used within LifeCLEF 2014 plant identification challenge was employed. One of the main outcomes of the experiment is that machines are still far from beating the best expert botanists. However, the best machine runs are competing with experienced botanists and do clearly outperform beginners and inexperienced test subjects. This shows that the performances of automated plant identification systems are very promising and might open the door to a new generation of ecological surveillance systems.

The next paper deals with "Point-based Medialness 2D Shape Description and Identification" (10.1007/s11042-015-2605-6). In this work, a perception-based medial point description of a natural form as a framework for a shape representation is proposed, which can then be efficiently used in biological species identification and matching tasks. Also, a robust shape matching algorithm is designed that finds the most relevant targets from a database of templates by comparing feature points in a scale, rotation and translation invariant way. The performance of the proposed method is tested on several databases.

The next paper applies the multimedia analysis and retrieval techniques in the water landscape. Specifically it deals with "Fine-Grained Object Recognition in Underwater Visual Data" (10.1007/s11042-015-2601-x) and focuses on determining fish species in low-quality image and video data. In this work a label propagation method is presented, which is able to transfer the labels from an annotated fish dataset to a set of 20 million fish images in order to achieve variability in fish appearance.

Finally, we present a very interesting application, which deals with seismic damage assessment. In this context a "An image-based seismic damage assessment system" (10.1007/s11042-015-2602-9) called IDEAS is proposed, to evaluate seismic damage inside buildings. IDEAS first compares images taken inside a building before and after an earthquake and it then maps the damage to a Mercalli intensity scale. In order to investigate the effectiveness and accuracy of IDEAS, over forty pairs of closedcircuit television (CCTV) images from Youtube website are used.

These seven papers deal with very interesting environmental multimedia retrieval challenges and therefore they are appealing to both the experts in the field, as well as to those who wish a snapshot of the current breadth of environmental multimedia retrieval research.

\section{References}

1. Graves N, Newsam S (2012) Visibility cameras: where and how to look, Proc. of the 1st ACM international workshop on Multimedia analysis for ecological data (7-12), Nara, Japan.

2. Joly A, Goëau H, Bonnet P, Bakić V, Barbe J, Selmi S et al (2013) Interactive plant identification based on social image data. Ecol Inform 23:22-34

3. Moumtzidou A, Epitropou V, Vrochidis S, Karatzas K, Voth S, Bassoukos A, Moßgraber J, Karppinen A, Kukkonen J, Kompatsiaris I (2013) A model for environmental data extraction from multimedia and its evaluation against various chemical weather forecasting datasets. Ecol Inform. doi:10.1016/j.ecoinf.2013.08. 003

4. Palazzo S, Spampinato C, Beyan C (2013) Event detection in underwater domain by exploiting fish trajectory clustering. In: Proc. of the IEEE ICIP 2013, Melbourne, Australia

5. Wanner L, Vrochidis S, Rospocher M, Mossgraber J, Bosch et al. (2012) Personalized environmental service orchestration for quality life improvement, 3rd Intelligent Systems for Quality of Life information Services Workshop (ISQL 2012), Halkidiki, Greece 


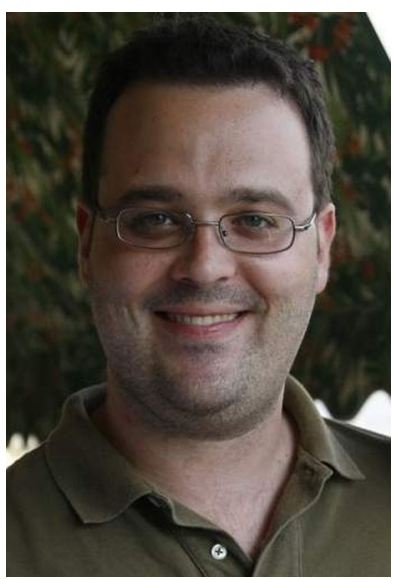

Stefanos Vrochidis Dr. Stefanos Vrochidis received the Diploma degree in Electrical Engineering from Aristotle University of Thessaloniki, Greece, the MSc degree in Radio Frequency Communication Systems from University of Southampton and the PhD degree in Electronic Engineering from Queen Mary University of London. Currently, he is a Researcher with the Information Technologies Institute. His research interests include semantic multimedia analysis, indexing and retrieval, search engine and human interactions as well as environmental and security applications. Dr. Vrochidis has successfully participated in many European and National projects such as PESCaDO and PEOPLE (as subcontractor) dealing with analysis and retrieval of environmental multimedia information. Currently Dr. Vrochidis is the Scientific Manager and Deputy Coordinator of FP7 STREP MULTISENSOR. He has been involved as a co-author in more than seventy related scientific journal, conference and book chapter publications. Dr. Vrochidis has served as a reviewer in international Journals such as Ecology Informatics, Multimedia Tools and Applications and as Technical program committee and reviewer in well reputed conferences and workshops such as ACM International Workshop on Multimedia Analysis for Ecological Data (MAED), European Conference on Information Retrieval (ECIR), ACM International Conference on Multimedia Retrieval (ICMR) and IEEE International Conference on Image Processing (ICIP).

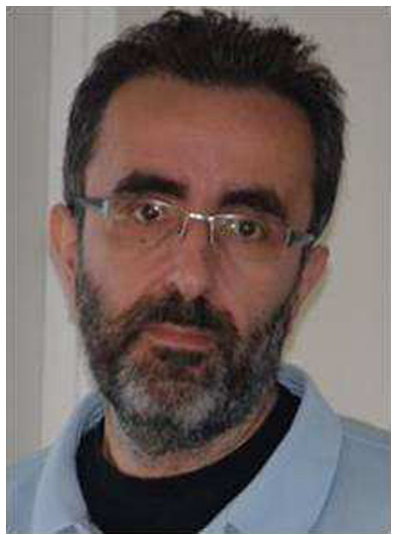

Kostas Karatzas Assoc. Professor Kostas Karatzas, Dr.-Eng. holds a Diploma and a Doctor degree in Mechanical Engineering, and leads the Informatics Systems and Applications-Environmental 
Informatics Research Group at the Dept. of Mechanical Engineering, where he teaches Informatics, Environmental Informatics and Environmental Impact Assessment. He is also teaching/has taught in various EU universities in MSc and seminar level, while he has been a visiting professor for the Finnish Meteorological Institute (FMI). Dr. Karatzas research work focuses mainly in informatics applications and environmental informatics, urban environment management and information systems, environmental (\& multimedia) data analysis and forecasting with the aid of computational intelligence methods and mathematical models, multimedia information content tools, and participatory environmental sensing. Dr. Karatzas has participated in more than 30 European R\&D projects, is Member of the International Scientific Advisory Board (SAB) of CLEEN's Measurement, Monitoring and Environmental Efficiency Assessment (MMEA) research programme, a member of the International Environmental Modelling and Software Society (iEMSs) Board of Directors, and an Associate Member of the OGC. He has authored approx 200 scientific papers, has been a member of the scientific committee of the many Environmental Informatics and Computational Intelligence conferences, and has/is supervising a number of $\mathrm{PhD}$ and MSc thesis in the area of Environmental Informatics-environmental information analysis and modelling.

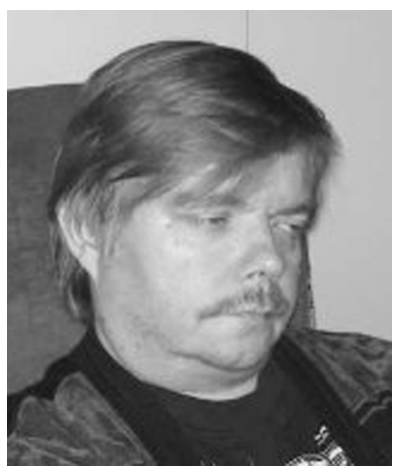

Ari Karppinen Adj. Prof. Ari Karppinen has received his degree of Doctor of Technology in Applied Physics at the HelsinkiUniversity of Technology in 2001 and was appointed as Adjunct Professor (Docent) in Physics at the University of Helsinki in 2004. His MSc thesis (1987) dealt with the description and application of a system for calculating radiation doses due to long range transport of radioactive releases and his Licentiates's thesis (1998) studied the effective choice of NOx - emission control measures. His doctor's thesis (2001) dealt with the meteorological pre-processing and atmospheric dispersion modelling of urban air quality and applications in the Helsinki metropolitan area. He has worked as a research scientist at the Finnish Meteorological Institute (FMI) since 1984. His expertise is on mathematical modelling, atmospheric physics and chemistry; particularly the evaluation of urban air quality and exposure and the dispersion of pollution from traffic. He is currently leading a research group (20 researchers) on Atmospheric Dispersion Modelling. The research group is working on 16 internationally funded (EU, ESA, ESF) and 10 nationally funded research and networking projects. The key areas of research involve modelling of urban air quality and exposure, regional and long-range transport of pollutants, accidents involving hazardous and radioactive materials and integration of meteorological models and measurements with the air quality modelling systems. He is the author of more than 270 scientific publications; 61 of these in refereed international journals. He has been working in 19 EU-funded projects, including vice-coordinator position in EU projectMARQUIS and scientific and technical coordination of recently finished EU/PESCaDO project. 


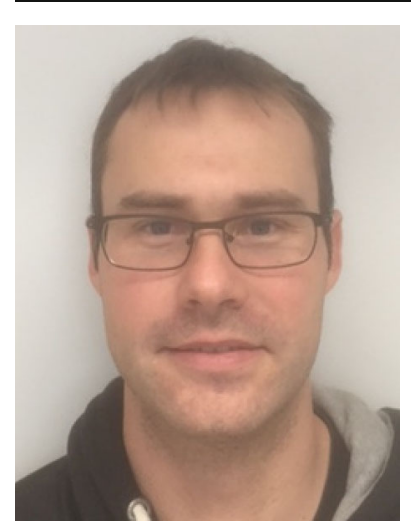

Alexis Joly is a permanent researcher at INRIA working on big multimedia data analytics and image search issues. He received his $\mathrm{PhD}$ degree in Computer Science in 2005 from the University of La Rochelle and his HDR degree in 2015 from the University of Montpellier. In 2007 and 2008, he coorganized CIVR and TRECVID video copy detection evaluation campaigns (NIST). He was involved in the steering board of several European projects (MUSCLE NoE, VITALAS IP, GLOCAL IP, CHORUS+ CA) and many national initiatives related to audiovisual archives, web user generated contents or biodiversity multimedia data. He is currently the scientific chair of the Pl@ntNet platform and the main organizer of the LifeCLEF international initiative. 